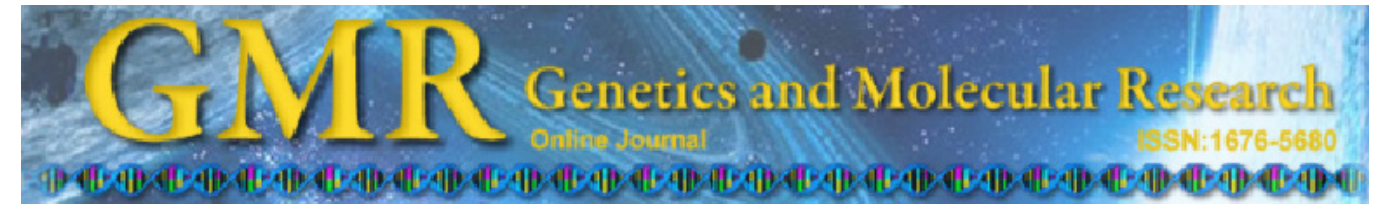

\title{
Mutational analyses of the TSC1 and TSC2 genes in cases of tuberous sclerosis complex in Chinese Han children
}

\author{
G.-X. Wang ${ }^{1,2}$, D.-W. Wang ${ }^{3}$, C.-Y. Yi ${ }^{2}$, J.-S. Qu$^{2}$ and Y.-L. Wang ${ }^{1}$ \\ ${ }^{1}$ Department of Paediatrics, \\ Provincial Hospital Affiliated to Shandong University, Jinan, Shandong, China \\ ${ }^{2}$ Medical Institute of Paediatrics, Qilu Children's Hospital, \\ Shandong University, Jinan, Shandong, China \\ ${ }^{3}$ School of Life Science, Shandong Normal University, Jinan, Shandong, China \\ Corresponding author: Y-L. Wang \\ E-mail: wangylsd@163.com
}

Genet. Mol. Res. 12 (2): 1168-1175 (2013)

Received July 10, 2012

Accepted October 11, 2012

Published April 12, 2013

DOI http://dx.doi.org/10.4238/2013.April.12.3

\begin{abstract}
Tuberous sclerosis complex (TSC) is an autosomal dominant neurogenetic disorder characterized by hamartomas in multiple organs and is caused by a wide spectrum of mutations in 1 of 2 causative genes (TSC1 or TSC2). Here, we present mutational analyses of the TSC1 and TSC2 genes in 4 cases of TSC in Chinese Han children, including 2 familial and 2 sporadic cases, using PCR and DNA sequencing of the entire coding region as well as exon-intron boundaries of these genes. Three mutations were identified in the TSC2 gene. Of these mutations, 2 mutations (c.3312-3313delGA and c.45delT) were novel, and the 3rd mutation (c.5238-5255del) was previously reported in Chinese Han and other populations. These mutations were not present in healthy family members or in 100 unrelated normal controls. The identification of these mutations in this study further expands the spectrum of known TSC2 gene mutations and contributes to prenatal molecular diagnosis and preimplantation genetic testing of TSC.
\end{abstract}

Key words: Tuberous sclerosis complex; TSC1 gene; TSC2 gene; Mutation 


\section{INTRODUCTION}

Tuberous sclerosis complex (TSC; MIM \#191100 and MIM \#613254) is an autosomal dominant neurogenetic disorder affecting 1/6000 live births. The main clinical features of this disease include seizure, mental retardation, and development of hamartomas in a variety of organs and tissues such as the skin, brain, heart, kidney, and liver (Choi et al., 2006; Mozaffari et al., 2009; Orlova and Crino, 2010; Jentarra et al., 2011). Two causative genes (TSC1 and TSC2) responsible for TSC have been identified, and both of these genes may be tumor suppressors (The European Chromosome 16 Tuberous Sclerosis Consortium, 1993; van Slegtenhorst et al., 1997). The human TSC1 gene on chromosome 9q34 consists of 23 exons and encodes a 130-kDa protein, TSC1 (or hamartin), and this protein spans 1164 amino acids (van Slegtenhorst et al., 1997). TSC1 has the following regions: a putative transmembrane domain composed of amino acids 127-144; a coiled-coil domain (CCD) spanning amino acids 719-998, which is necessary for protein-protein interactions between TSC1 and TSC2; a rho-activating domain comprised of amino acid residues 145-510, which activates rho GTPase; and amino acid residues 881-1084 that interact with the $\mathrm{N}$-terminus of the ezrin-radixin-moezin family of actin-binding proteins (Napolioni and Curatolo, 2008). The TSC2 gene, which is located on chromosome 16p13.3 contains 41 exons and encodes a 200-kDa protein with 1807 amino acids; TSC2 or tuberin contains the following 7 functional domains, from the $\mathrm{N}$-terminus to the C-terminus: leucine zipper domain (LZD) of amino acids 81-98 encoded by exon 3; CCD1 of amino acids 346-371 encoded by exon 10; CCD2 of amino acids 1008-1021 encoded by exon 26; transcription activation domain 1 (TAD1) of amino acids 1163-1259 encoded by exons 29-30; GTPase-activating protein domain (GAPD) of amino acids 1517-1674 encoded by exons 34-38; TAD2 of amino acids 1690-1744 encoded by exons 39-40; and a calmodulin-binding domain (CaMD) of amino acids 1740-1755 encoded by exons 40-41 (Povey et al., 1994; Krymskaya, 2003; Napolioni and Curatolo, 2008). TSC1 and TSC2 form a complex that activates the GTPase activity of rheb, preventing the rhebGTP-dependent stimulation of cell proliferation, adhesion, growth, differentiation, and migration through mTOR (Garami et al., 2003; Hung et al., 2006). The molecular basis of TSC is impairment of the TSC1/TSC2 complex (Huang and Manning, 2008; Mozaffari et al., 2009). Approximately two-thirds of TSC cases are sporadic and appear to result from de novo germline mutations in both TSC genes (www.hgmd.cf.ac.uk/ac/gene.php?gene=TSC1; www.hgmd.cf.ac.uk/ac/ gene.php?gene=TSC2). The present report summarizes recent results of our ongoing efforts to identify TSC1 and TSC2 mutations in Chinese Han children with TSC (Wang et al., 2011).

\section{MATERIAL AND METHODS}

\section{Patients}

One male and 3 female probands or patients with TSC were enrolled in this study. These children were from different families, and included 2 familial and 2 sporadic cases. All patients fulfilled the definitive TSC criteria of the 1998 Tuberous Sclerosis Consensus Conference (Roach et al., 1998).

All subjects belong to the Chinese Han ethnic group; their standardized clinical data are based on a detailed clinical evaluation comprising medical history and physical examination. This study was approved without restrictions by the Research Ethics Committee of a Provincial Hospital Affiliated to Shandong University, and consent was obtained by all subjects or their parents. 


\section{DNA isolation and mutation analysis}

Blood ( $3 \mathrm{~mL}$ ) was drawn from the probands, their affected relatives, their healthy family members, and 100 unrelated normal controls who had no physical/psychological diseases and whose first-degree relatives had no history of TSC. Genomic DNA was extracted from blood samples using the TIANamp Blood DNA Kit (Tiangen Biotech Co., Beijing, China). Amplicons were then obtained with 25 pairs of PCR primers for exons 1 to 23 of the TSC1 gene and 36 pairs of PCR primers for exons 0 to 41 of the TSC2 gene; the primers spanned the entire coding region as well as the exon-intron boundaries of these genes. The sequences of the primers are listed in our previous study (Wang et al., 2011).

The PCR was performed using a GeneAmp 9700 thermal cycler (Perkin-Elmer, Waltham, MA, USA) with $2 X$ Hotstart Taq PCR Mastermix (Tiangen Biotech). In a 50- $\mu \mathrm{L}$ reaction mixture, $300 \mathrm{ng}$ genomic DNA was used with $2.0 \mu \mathrm{L} 10 \mu \mathrm{M}$ of each primer and $25 \mu \mathrm{L} 2 \mathrm{X}$ PCR Mastermix. Genomic DNA was first denatured at $94^{\circ} \mathrm{C}$ for $3 \mathrm{~min}$, followed by 31 cycles at $94^{\circ} \mathrm{C}$ for $35 \mathrm{~s}, 50^{\circ}$ to $62^{\circ} \mathrm{C}$ for $35 \mathrm{~s}$, and $72^{\circ} \mathrm{C}$ for $50 \mathrm{~s}$. The PCR products were finally extended at $72^{\circ} \mathrm{C}$ for $5 \mathrm{~min}$. The products were gel purified using agarose gel DNA purification kit (Tiangen Biotech), and the purified PCR products were sequenced using forward and reverse primers. Automated sequencing was performed on an ABI 377 sequencer. Each mutation was confirmed by sequencing of individual amplicons. If a mutation within an exon was detected in the proband and the relatives of the proband, this exon was PCR-amplified specifically from DNA from the healthy members of the proband family as well as from 100 unrelated normal controls.

\section{RESULTS}

\section{Patient characteristics} sporadic.

Of the 4 cases, cases 1 and 4 were familial (Figure 1a,b), and cases 2 and 3 were

Case 1 was a 14-year-old male. He had his first epileptic attack in June 2008 and showed facial angiofibromas (Figure 2a) and truncal white macules. A CT scan of the brain showed that he also had subependymal nodules along the lateral walls of the lateral ventricles. Pathological examination revealed subependymal giant cell astrocytoma in the anterior wall of the left lateral ventricle. This case was a familial case because the grandmother, father, and great aunt also had facial angiofibromas and subependymal nodules.

Case 2 was a 7-month-old female. She experienced seizures 3 times within 1 month and demonstrated a moderate developmental delay. Dermatological examination identified abdominal white macules. Subependymal calcifications were identified by a brain CT scan (Figure 2b). The electroencephalogram revealed a spike wave/spike-slow wave complex in the cortical region. Her parents and other family members were not affected with TSC.

Case 3 was a 2-year-old female. She received medical attention for frequent seizures during 1.5 years. She had white patches on her face, forearms, and back. Radiological examination of the brain showed multiple subependymal calcifications, and CT scans showed multiple cortical tubers. Her parents and a younger sister had no neurological and dermatological findings.

Case 4 was a 7-month-old female that had contracted West syndrome at the age of 5 months. Physical examination showed that hypomelanotic macules were present on her back and hips. Subependymal nodules were identified in both sides of the lateral ventricle by a brain 
CT scan. Her mother had the same dermatological and radiological findings. The clinical features of these cases are summarized in Table 1.

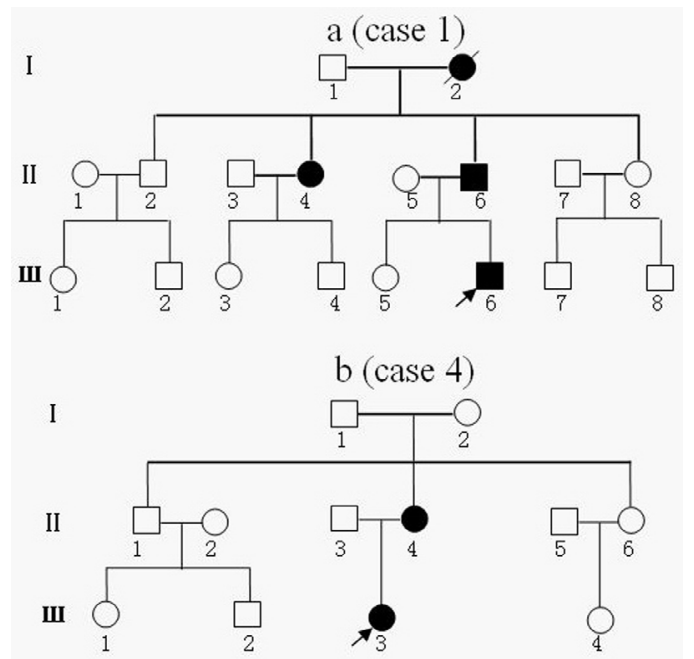

Figure 1. Pedigrees of two familial cases with tuberous sclerosis complex. Filled circles and squares represent affected females and males, respectively; proband is indicated with an arrow.
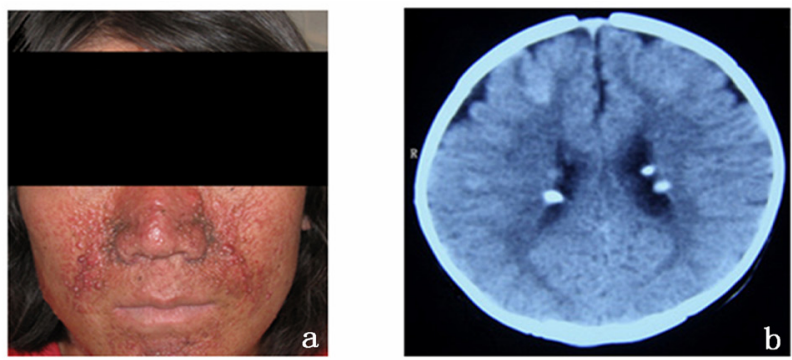

Figure 2. Major features of the patients with tuberous sclerosis complex. a. Angiofibromas on the face of the proband of case 1. b. Cranial CT scan of case 2: subependymal calcifications along the lateral walls of the lateral ventricles.

Table 1. Clinical features of four cases of tuberous sclerosis complex.

\begin{tabular}{|c|c|c|c|c|c|c|}
\hline Patient ID & FA & HM & $\mathrm{SZ}$ & SEN & SEGA & $\mathrm{AL}$ \\
\hline \multicolumn{7}{|l|}{ Case 1 (f) } \\
\hline$\alpha 4$ & + & + & + & + & - & - \\
\hline$\alpha 6$ & + & + & - & + & - & + \\
\hline$\beta 6$ & + & + & + & + & + & + \\
\hline Case $2(\mathrm{~s})$ & - & + & + & + & - & - \\
\hline Case 3 (s) & - & + & + & + & - & - \\
\hline \multicolumn{7}{|l|}{ Case 4 (f) } \\
\hline$\alpha 4$ & + & - & - & + & - & - \\
\hline$\beta 3$ & - & + & + & + & - & - \\
\hline
\end{tabular}

$\mathrm{f}=$ familial; $\mathrm{s}=$ sporadic FA = facial angiofibromas; $\mathrm{HM}=$ hypomelanotic macules; $\mathrm{SZ}=$ seizures; $\mathrm{SEN}=$ subependymal nodule; SEGA = subependymal giant cell astrocytoma; $\mathrm{AL}=$ angiomyolipomas; $(+)=$ symptom present; $(-)=$ symptom absent. 


\section{Sequence analysis}

We analyzed the entire coding region and the exon-intron boundaries of TSC1 and TSC2 in 4 familial and sporadic cases of TSC using PCR and DNA sequencing. Three mutations were identified in the TSC2 gene, but no mutation was found in the TSC1 gene. Of these mutations, a deletion mutation in exon 28 (c.3312-3313delGA) (Figure 3A) and exon 1 (c.45delT) (Figure 3B) in cases 2 and 4, respectively, were novel, and the 3rd mutation in exon 40 (c.5238-5255del) (Figure 3C), detected in case 1, had previously been reported in Chinese Han and other populations. None of these mutations was observed in healthy family members of the patients or 100 unrelated normal controls, suggesting that the sequence aberration was pathogenic. Table 2 summarizes the mutations identified in this study.
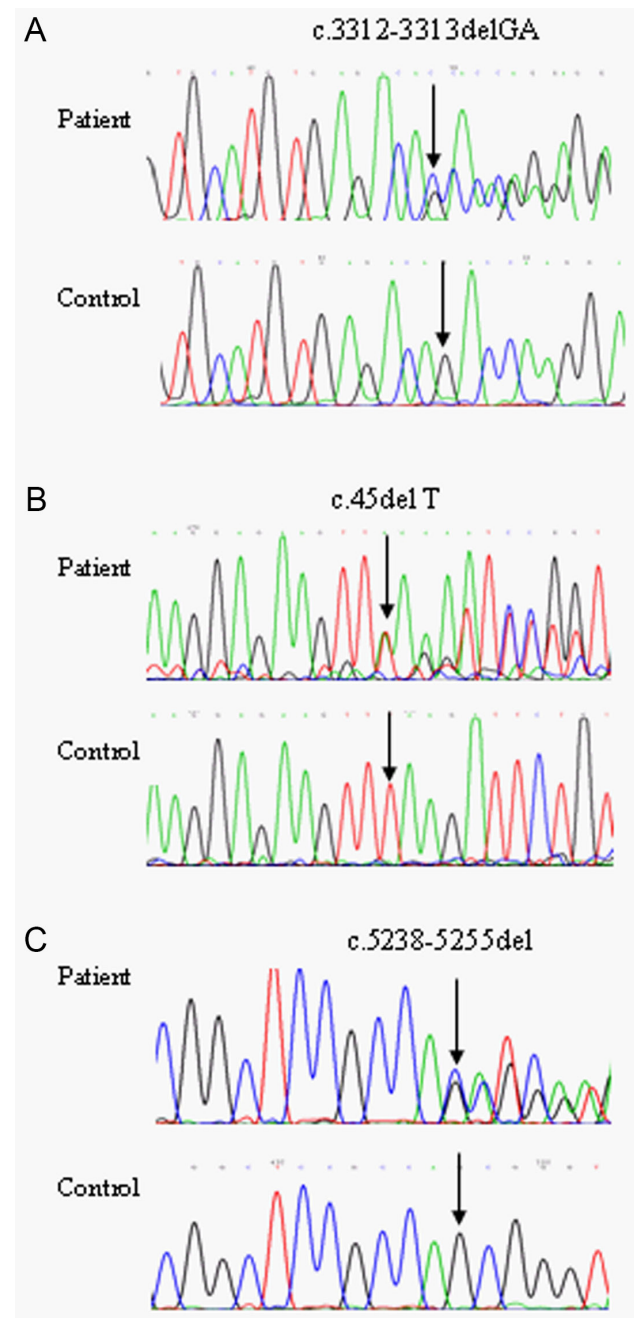

Figure 3. Sequence chromatograms of the TSC2 gene in index patients and controls. Arrows indicate the sites of mutations in the probands from cases $2,4,1$, respectively $(\mathbf{a}, \mathbf{b}, \mathbf{c})$, or the corresponding sites in controls. 


\begin{tabular}{|c|c|c|c|c|c|}
\hline & Location & Nucleotide change & Amino acid change & Type & New or reported \\
\hline Case 1 (f) & Exon 40 & c.5238-5255del & His $1746 \mathrm{Gln} / 1747-1752 \mathrm{del}$ & Sub/del & Reported \\
\hline Case 2 (s) & Exon 28 & c.3312-3313delGA & His $1104 f \mathrm{f} / 1166 \mathrm{X}$ & Frameshift & New \\
\hline Case 4 (f) & Exon 1 & c.45delT & Leu15fs/45X & Frameshift & New \\
\hline
\end{tabular}

$\mathrm{s}=$ sporadic case $; \mathrm{f}=$ familial case. $\mathrm{Sub} / \mathrm{del}=$ substitution with deletion.

\section{DISCUSSION}

TSC is characterized by hamartomas in multiple organs, including the brain, skin, heart, kidneys, and lungs. Central nervous system manifestations include epilepsy, autism, and behavioral problems. Renal lesions, usually angiomyolipomas, can cause renal failure. Skin lesions include melanotic macules, facial angiofibromas, and patches of connective tissue nevi. The diagnosis of TSC is performed based on the above clinical manifestations, which are categorized into major and minor features. The presence of 2 major features, or 1 major and 2 minor features, is sufficient for definitive diagnosis (Roach et al., 1998). All 4 patients had at least 2 major features: hypomelanotic macules, angiofibromas, or subependymal nodules, thus providing a definitive diagnosis of TSC.

The mutation spectrum of the TSC genes is highly heterogeneous. More than 200 unique TSC1 mutations and 700 unique TSC2 mutations have been reported. In this study, we screened 4 TSC cases for mutations in all coding regions and exon-intron boundaries of the TSC1 and TSC2 genes, and 3 causative mutations in the TSC2 gene were detected in these cases, with a mutation rate of $75 \%$, similar to that obtained previously (Ali et al., 2005; Camposano et al., 2009). van Slegtenhorst et al. (1997) reported that approximately 10 to $30 \%$ of all TSC cases are due to mutations in the TSC1 gene, but no mutation was found in the TSC1 gene in our study.

Of the 3 mutations in the present study, 2 deletion mutations in exon 28 (c.33123313 delGA) and exon 1 (c.45delT) of the TSC2 gene were novel; the 3 rd mutation was an 18-bp deletion in exon 40 of the TSC2 gene and was previously reported in Chinese Han and Caucasian populations (Beauchamp et al., 1998; Yao et al., 2008). The 18-bp deletion, which results in the substitution of a glutamine for a histidine at codon 1746, with the removal of codons 1747 through 1752, is in the CaMD domain of TSC2. Noonan et al. (2002) found that the expression of the 6 amino acid in-frame deletion significantly repressed ligand-induced steroid/nuclear receptor-mediated transcription activity, leading to uncontrolled cell growth and cell proliferation and consequent malformations consisting of disorganized arrangement of tissue types, such as hypomelanotic macules, angiofibromas, and subependymal nodules. The c.3312delGA mutation in TSC2 is predicted to truncate the TSC2 protein by 704 amino acids and add 62 amino acids, resulting in an alternative protein that lacks 4 of the 7 domains: TAD1, GAPD, TAD2, and CaMD. The c.45delT mutation in the TSC2 gene is a frame-shift mutation, resulting in 30 missense amino acids followed by a premature stop codon, resulting in a small protein of 44 amino acids instead of the normal TSC2 protein of 1807 amino acids, lacking all 7 domains: LDZ, CCD1, CCD2, TAD1, GAPD, TAD2, and CaMD. After searching the SNP database and the human gene mutation database, we found that c.3312delGA and c.45delT in the TSC2 gene are absent from the 2 databases. Because of the 2 new mutations in the TSC 2 gene, one or more functional domains are likely lacking, explaining the TSC phenotype. 
We did not find any mutation in the TSC1 and TSC2 genes in case 3, and the number of detected pathogenic mutations is probably underestimated because there may be mutations in regions that have not been analyzed (e.g., introns, 3'- and 5'-untranslated regions, and distant enhancers) or large insertions, deletions, and rearrangements that cannot be detected using conventional PCR techniques. Therefore, a suitable advanced methodology, such as multiplex ligation-dependent probe amplification, should be used to detect mutations.

In summary, the PCR and DNA sequence analyses identified three mutations in the TSC2 gene in 4 sporadic and familial TSC cases in Chinese Han children. Of these mutations, 2 mutations are new. The identification of these 2 mutations further expands the mutation spectrum of the TSC 2 gene and contributes to prenatal molecular diagnosis and preimplantation genetic testing of TSC.

\section{ACKNOWLEDGMENTS}

The authors gratefully acknowledge the patients with TSC for their participation and cooperation in this study. The authors also thank Dr. Guangfang Ren for manuscript revisions.

\section{REFERENCES}

Ali M, Girimaji SC, Markandaya M, Shukla AK, et al. (2005). Mutation and polymorphism analysis of TSC1 and TSC2 genes in Indian patients with tuberous sclerosis complex. Acta Neurol. Scand. 111: 54-63.

Beauchamp RL, Banwell A, McNamara P, Jacobsen M, et al. (1998). Exon scanning of the entire TSC2 gene for germline mutations in 40 unrelated patients with tuberous sclerosis. Hum. Mutat. 12: 408-416.

Camposano SE, Greenberg E, Kwiatkowski DJ and Thiele EA (2009). Distinct clinical characteristics of tuberous sclerosis complex patients with no mutation identified. Ann. Hum. Genet. 73: 141-146.

Choi JE, Chae JH, Hwang YS and Kim KJ (2006). Mutational analysis of TSC1 and TSC2 in Korean patients with tuberous sclerosis complex. Brain Dev. 28: 440-446.

Garami A, Zwartkruis FJ, Nobukuni T, Joaquin M, et al. (2003). Insulin activation of Rheb, a mediator of mTOR/S6K/4EBP signaling, is inhibited by TSC1 and 2. Mol. Cell 11: 1457-1466.

Huang J and Manning BD (2008). The TSC1-TSC2 complex: a molecular switchboard controlling cell growth. Biochem. J. 412: 179-190.

Hung CC, Su YN, Chien SC, Liou HH, et al. (2006). Molecular and clinical analyses of 84 patients with tuberous sclerosis complex. BMC Med. Genet. 7: 72.

Jentarra GM, Rice SG, Olfers S, Saffen D, et al. (2011). Evidence for population variation in TSC1 and TSC2 gene expression. BMC Med. Genet. 12: 29.

Krymskaya VP (2003). Tumour suppressors hamartin and tuberin: intracellular signalling. Cell Signal. 15: 729-739.

Mozaffari M, Hoogeveen-Westerveld M, Kwiatkowski D, Sampson J, et al. (2009). Identification of a region required for TSC1 stability by functional analysis of TSC1 missense mutations found in individuals with tuberous sclerosis complex. BMC Med. Genet. 10: 88.

Napolioni V and Curatolo P (2008). Genetics and molecular biology of tuberous sclerosis complex. Curr. Genomics 9: 475-487.

Noonan DJ, Lou D, Griffith N and Vanaman TC (2002). A calmodulin binding site in the tuberous sclerosis 2 gene product is essential for regulation of transcription events and is altered by mutations linked to tuberous sclerosis and lymphangioleiomyomatosis. Arch. Biochem. Biophys. 398: 132-140.

Orlova KA and Crino PB (2010). The tuberous sclerosis complex. Ann. N. Y. Acad. Sci. 1184: 87-105.

Povey S, Burley MW, Attwood J, Benham F, et al. (1994). Two loci for tuberous sclerosis: one on 9q34 and one on 16p13. Ann. Hum. Genet. 58: 107-127.

Roach ES, Gomez MR and Northrup H (1998). Tuberous sclerosis complex consensus conference: revised clinical diagnostic criteria. J. Child Neurol. 13: 624-628.

The European Chromosome 16 Tuberous Sclerosis Consortium (1993). Identification and characterization of the tuberous sclerosis gene on chromosome 16. Cell 75: 1305-1315. 
van Slegtenhorst M, de Hoogt R, Hermans C, Nellist M, et al. (1997). Identification of the tuberous sclerosis gene TSC1 on chromosome 9q34. Science 277: 805-808.

Wang GX, Wang DW, Zhao JS, Wang SF, et al. (2011). A novel TSC1 mutation (c.1964delA) in a Chinese patient with tuberous sclerosis complex. Genet. Mol. Res. 10: 107-113.

Yao FM, Fan X, Zhou FS, Quan H, et al. (2008). Analysis of gene mutations in two Chinese patients with tuberous sclerosis. Zhongguo Ma Feng Pi Fu Bing Za Zhi 24: 861-863. 